\title{
Two phase collective modes in a Josephson vortex lattice in the intrinsic Josephson junction $\mathrm{Bi}_{2} \mathrm{Sr}_{2} \mathrm{CaCu}_{2} \mathrm{O}_{8+\delta}$
}

\author{
I. Kakeya,* T. Wada, R. Nakamura, and K. Kadowaki \\ Institute of Materials Science, University of Tsukuba, Tsukuba, Ibaraki 305-8573, Japan \\ (Received 29 March 2005; revised manuscript received 24 May 2005; published 27 July 2005)
}

\begin{abstract}
Josephson plasma excitations in the high- $T_{c}$ superconductor $\mathrm{Bi}_{2} \mathrm{Sr}_{2} \mathrm{CaCu}_{2} \mathrm{O}_{8+\delta}$ have been investigated in a wide microwave frequency region $(9.8-75 \mathrm{GHz})$ - in particular, in a magnetic field applied parallel to the $a b$ plane of the single crystal. In sharp contrast to the case for magnetic fields parallel to the $c$ axis or tilted from the $a b$ plane, it was found that there are two kinds of resonance modes, which are split in energy and possess two distinctly different magnetic field dependences. One always lies higher in energy than the other and has a shallow minimum at about $0.8 \mathrm{kOe}$, then increases linearly with magnetic field. On the other hand, another mode begins to appear only in a magnetic field (from a few kOe and higher) and has a weakly decreasing tendency with increasing magnetic field. By comparing with a recent theoretical model the higher-energy mode can naturally be attributed to the Josephson plasma resonance mode propagating along the primitive reciprocal lattice vector of the Josephson vortex lattice, whereas the lower-frequency mode is assigned to the novel phase collective mode of the Josephson vortex lattice, which has never been observed before.
\end{abstract}

DOI: 10.1103/PhysRevB.72.014540 PACS number(s): 74.25.Qt, 74.50.+r, 74.25.Nf, 72.30.+q

\section{INTRODUCTION}

The phase collective excitation in Josephson junctions known as Josephson plasma has been studied since the 1960s, triggered by a theoretical prediction of Anderson. ${ }^{1}$ However, there are only a few experimental reports on Josephson plasma resonance in single junctions made by conventional superconductors ${ }^{2,3}$ because there is a strong damping of the quasiparticles due to the small superconducting energy gap in the single junctions. This situation was improved drastically in the case of high- $T_{c}$ superconductors because they are comprised of a well-established weakly coupled Josephson junctions along the crystallographic $c$ axis (intrinsic Josephson junction), whose plasma gap $\hbar \omega_{p}$ along the $c$ axis is much lower than the superconducting gap. In particular, in the case of $\mathrm{Bi}_{2} \mathrm{Sr}_{2} \mathrm{CaCu}_{2} \mathrm{O}_{8+\delta}(\mathrm{Bi} 2212)$, the superconducting $\mathrm{CuO}_{2}$ layers and the insulating or semiconducting $\mathrm{Bi}_{2} \mathrm{O}_{2}$ layers are regularly stacked in an atomic level in the unit cell. ${ }^{4,5}$ Based on this, Tachiki and co-workers predicted that the Josephson plasma excitation is well defined in high- $T_{c}$ superconductors. ${ }^{6,7}$ Subsequently, two theoretical models have been developed: one by Koyama and Machida $^{8,9}$ and the other by Pedersen and Sakai. ${ }^{10,11}$ Quite recently, a unified theory has been reported. ${ }^{12}$

The Josephson plasma resonance in Bi2212 is observed as a sharp and strong microwave absorption in a finite magnetic field parallel to the $c$ axis. ${ }^{13}$ Especially, the longitudinal Josephson plasma mode gives an extremely sharp resonance because of its small dispersion and the high quasiparticle damping rate of Bi2212. ${ }^{14,15}$ The clear observation of the longitudinal plasma resonance has been a strong advantage of Bi2212 for investigation of the Josephson plasma phenomena in superconductors compared with many other materials such as $\mathrm{La}_{2-x} \mathrm{Sr}_{x} \mathrm{CuO}_{4},{ }^{16} \quad \mathrm{Bi}_{2}(\mathrm{Sr}, \mathrm{La})_{2} \mathrm{CuO}_{6+\delta}$ $(\mathrm{Bi} 2201),{ }^{17} \mathrm{YBa}_{2} \mathrm{Cu}_{3} \mathrm{O}_{7-\delta} \quad(\mathrm{YBCO}),{ }^{18}$ and BEDT-TTF salts, ${ }^{19}$ in which the Josephson plasma resonance (edge) is less clear.
According to the theory of the Josephson plasma resonance, the resonance frequency $\omega_{p}(H, T)$ can be written as ${ }^{7,20}$

$$
\omega_{p}^{2}(H, T)=\omega_{p}^{2}(T)\left\langle\cos \varphi_{l, l+1}(H, T)\right\rangle,
$$

where $\omega_{p}(T)=c / \sqrt{\epsilon} \lambda_{c}(T)$ is the Josephson plasma frequency in the absence of a magnetic field. $\epsilon$ and $\lambda_{c}(T)$ stand for the dielectric constant and the temperature-dependent $c$ axis penetration depth, respectively. $\varphi_{l, l+1}(H, T)$ is the gaugeinvariant phase difference between the $l$ th and $(l+1)$ th layers, and $\langle\cdots\rangle$ denotes the spatial and time averages.

In the case of a magnetic field parallel to the $c$ axis (perpendicular to the superconducting $\mathrm{CuO}_{2}$ layers), it is well known that pancake vortices are generated above $H_{c 1}$, the lower critical field. For the Josephson plasma resonance, this situation is taken into account by considering the uniform reduction of the Josephson current along the $c$ axis, resulting in the decrease of $\left\langle\cos \varphi_{l, l+1}(H, T)\right\rangle$-i.e., the reduction of $\omega_{p}(H, T)$ in Eq. (1). Because of this fact, the Josephson plasma resonance can be used as a sensitive method to evaluate $\left\langle\cos \varphi_{l, l+1}(H, T)\right\rangle .{ }^{21-24}$

This treatment cannot be applied to the case where the external magnetic field is parallel to the $a b$ plane. In this case, the Josephson vortices (JV's) are introduced in between the $\mathrm{CuO}_{2}$ double layers and contribute the Josephson plasma resonance as an essential ingredient of the phenomenon. In contrast to the case for the perpendicular field, the plasma frequency is hardly suppressed because the fluctuations of pancake vortices are too small to dominate the interlayer coupling. This is clearly observed in the angular dependence of the Josephson plasma resonance near the $a b$ plane. A sharp dip in the Josephson plasma resonance field is clearly observed within an angle of $\pm 2^{\circ}$ from the $a b$ plane. ${ }^{25}$ From this sharp change of the Josephson plasma resonance behavior, it is in turn expected that the dynamical motion of JV's such as JV lattice collective modes has been thought to account for the Josephson plasma resonance frequency. This 
opens entirely new possibilities for the Josephson plasma resonance phenomena in the case of parallel magnetic field configurations and deserves more detailed consideration both experimentally and theoretically. Since the JV modulates the interlayer Josephson tunneling current in the length scale of $\lambda_{J} \equiv \gamma s$ along the $a b$ plane, where $\gamma$ is the anisotropy parameter and $s$ is the interlayer distance, the collective plasma oscillation strongly couples with the collective JV motion such as JV lattice modes.

This picture is initiated by the single-junction model, which was studied in the 1960 s by Lebwohl and Stephen ${ }^{26}$ and by Fetter and Stephen ${ }^{27}$ theoretically. They solved a onedimensional nonlinear wave equation and obtained two modes as excitation spectra: one of them lies above the plasma gap $\hbar \omega_{p}$ in zero field and approaches asymptotically the linear relation with the propagation vector $\boldsymbol{k}$ (plasma mode). Another mode obtained by Fetter and Stephen in a single junction is a gapless vortex sliding mode. The plasma mode with an array of Josephson vortices was first of all observed indirectly as a so-called Eck resonance in the current-voltage $(I-V)$ characteristics of the $\mathrm{Pb} / \mathrm{PbO} / \mathrm{Pb}$ single junction, in which the resonance was detected at a voltage proportional to the applied parallel field $H_{\|}{ }^{28}$ Fiske has also shown an anomalous steplike behavior in the $I-V$ characteristics in $\mathrm{Al}, \mathrm{Sn}, \mathrm{Pb}$, and $\mathrm{Nb}$ junctions, ${ }^{29}$ and this phenomenon was associated with the resonant electromagnetic modes of the junction.

More recently, Josephson plasma resonance experiments have been performed in the external magnetic field configuration being closely parallel to the $a b$ plane in single-crystal Bi2212. ${ }^{25,30,31}$ They observed anomalous behaviors such as the angular dependence of the resonance field associated with the sudden jump of it, which have partly been explained by the theory of Bulaevskii et al. ${ }^{32}$ It seems that the resonance mode indicating this anomalous behavior can be described qualitatively by the single-junction model. ${ }^{27}$ However, the model in which conventional (metallic) single junctions are connected in series cannot be applied in intrinsic Josephson junctions especially in treating Josephson plasma excitations, because the charge conservation inside a layer does not hold due to thinner $\mathrm{CuO}_{2}$ double layers $(3 \AA)$ than the charge screening length $(\mu \sim 10 \AA)$. Therefore, we consider that the previous treatment of the Josephson plasma resonance in a parallel magnetic field is insufficient and exclude the rich physics inherent to the intrinsic Josephson junctions.

In this paper, we report observation of two-phase collective modes through systematic measurements of microwave absorption as functions of magnetic fields parallel to the $a b$ plane, temperature, and frequency in Bi2212 single crystals, and then the origins of the two modes are argued. The organization is as follows. Experimental results by using the microwave resonance technique described in Sec. II are presented in Sec. III. In this section, we first present the experimental data at $25.5 \mathrm{GHz}$, where the phenomena are the most interesting and the richest among all frequencies (Sec. III A). Next, we show the experimental results at different frequencies and describe the frequency dependence in order to obtain a field dependence of the resonance modes (Sec. III B). Finally, universal features deduced from experimental results in crystals with different doping levels are described (Sec. III C). The origins of the observed two modes are discussed in Sec. IV with the help of recent theoretical models. ${ }^{33-35}$

\section{EXPERIMENTAL SETUP}

In order to perform Josephson plasma experiments in the JV state, it is necessary to have a wide range of microwave frequencies beyond the zero-field plasma frequency, which is expected above $100 \mathrm{GHz}$ for optimally doped Bi2212. Since in our experimental facility the microwave frequencies were limited up to $90 \mathrm{GHz}$, it was necessary to bring down the zero-field Josephson plasma resonance in the range of $50 \mathrm{GHz}$. This was achieved by shifting the doping level to the underdoped region by annealing the pristine overdoped Bi2212 samples under reduced atmospheres.

We have measured microwave absorption in three Bi2212 single crystals grown by the modified traveling-solvent floating-zone (TSFZ) method. Two underdoped crystals (U1, U2) and one optimally doped crystal (OP) were used in the measurements for comparison. The superconducting transition temperatures $T_{c}$ 's of these crystals were determined by low-field magnetization measurements with a superconducting quantum interference device (SQUID) magnetometer as 70.2, 76.8, and $90.5 \mathrm{~K}$ with superconducting transition widths of 1.0, 2.5, and $0.8 \mathrm{~K}$ for $\mathrm{U} 1, \mathrm{U} 2$, and $\mathrm{OP}$, respectively. The typical size of the crystals is $0.8 \times 0.8$ $\times 0.02 \mathrm{~mm}^{3}$.

Most of the microwave absorption data except for those in Sec. III C have been obtained in U1. Measurements were made in a frequency range between 9.8 and $75 \mathrm{GHz}$ by both reflection- and transmission-type bridge balance techniques using rectangular cavity resonators with $\mathrm{TE}_{102}$ mode. In order to excite the Josephson plasma, the samples were placed inside the cavity in such a way that the oscillating electric field of the microwaves was exerted parallel to the $c$ axis over the $a b$ plane of the crystal as shown in Fig. 1 of Ref. 36 . Frequency-stabilized microwaves were generated by the synthesized signal generator (Hewlett-Packard 83650B) or Gunn oscillators, and the magnetic field was applied by a split-pair superconducting magnet. The angle between field direction and $\mathrm{CuO}_{2}$ plane of the sample was adjusted by rotating the cavity resonator with respect to the field direction by a precision rotator within an accuracy of $0.001^{\circ}$.

The resonance data were obtained either by sweeping the magnetic field at various fixed the temperatures or by sweeping the temperature at various fixed fields. The resonance occurs when $\omega_{p}(H, T)$ matches with the incident microwave frequency $\omega$ by varying either the magnetic field or temperature. It is worth noting that the field sweep (FS) measurements would give a nonequilibrium JV state because the strong hysteretic behavior of the resonance was observed, especially at low temperatures while the magnetic field was swept up and down. In order to avoid this unnecessary complication, we employed measurements mostly by the temperature sweep (TS) when low-temperature data were needed. The temperature was either stabilized within $20 \mathrm{mK}$ during the field sweep or swept at a rate of $2 \mathrm{~K} / \mathrm{min}$. It is 


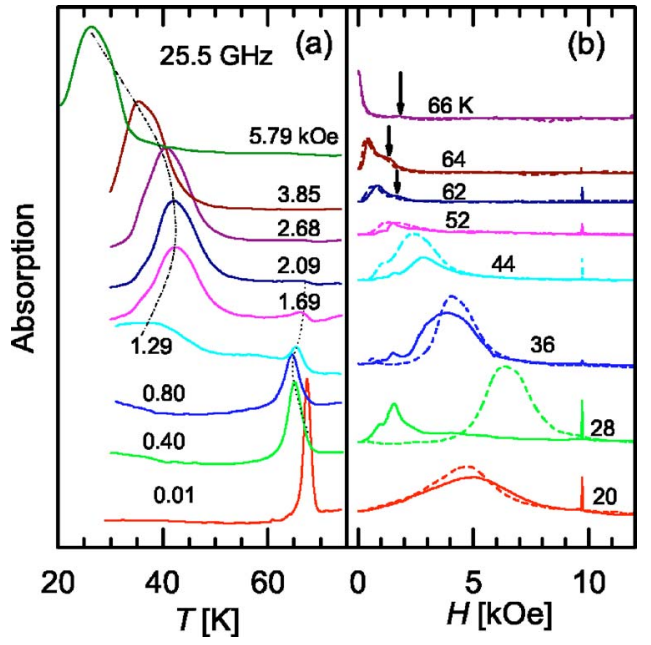

FIG. 1. (Color online) The resonance curves obtained at $25.5 \mathrm{GHz}$ by sweeping the temperature (a) and by sweeping the field (b). In (a), the temperature was swept from far above $T_{c}$ to the lowest temperature after changing magnetic field at each measurement, and no hysteresis was found. In (b), the solid and dashed curves indicate data obtained in increasing and decreasing fields. Hysteresis found in field sweep measurement becomes more significant at lower temperatures and in lower fields. Thick arrows in (b) denote shoulders attributed to the weak resonance of the HTM above the turnover. The sharp peak shown in (b) at $10 \mathrm{kOe}$ is due to DPPH (1,1-dyphenil-2-picryl hydragyl) as a field marker.

also worth noting that since it is technically difficult to sweep microwave frequency as far as the cavity resonator method is employed, we are compelled to prepare a number of cavities (about 15 cavities) with different resonance frequencies.

\section{EXPERIMENTAL RESULTS}

\section{A. Simultaneous observation of two resonance modes}

Figure 1(a) shows microwave absorption curves at $25.5 \mathrm{GHz}$ in various constant parallel magnetic fields $H_{\|}$obtained by TS measurements. Two clear resonance lines with different characters are found at higher and lower temperatures. As is seen clearly, there is a well-separated temperature gap in between. This feature strongly depends on the microwave frequencies: the higher in frequency, the larger separation in temperature is observed. As will be shown later, the lower-temperature mode (LTM) quickly shifts towards the low-temperature side and disappears with increasing microwave frequency $\omega$. On the other hand, with lowering frequency below $30 \mathrm{GHz}$, the LTM quickly shifts up and merges with the higher-temperature mode (HTM), which is explained in Fig. 2. In general, the HTM in this frequency region appears only in a relatively low field, approximately below $3 \mathrm{kOe}$, as long as $\omega$ is lower than $55 \mathrm{GHz}$, which is close to the zero-field and zero-temperature plasma frequency $\omega_{p}(0)$ described later. When $\omega$ goes beyond $33 \mathrm{GHz}$, only one resonance is observed as shown in Fig. 3. In the frequency region between 10 and $30 \mathrm{GHz}$ both resonances come into a play, resulting in a complicated phenomenon as

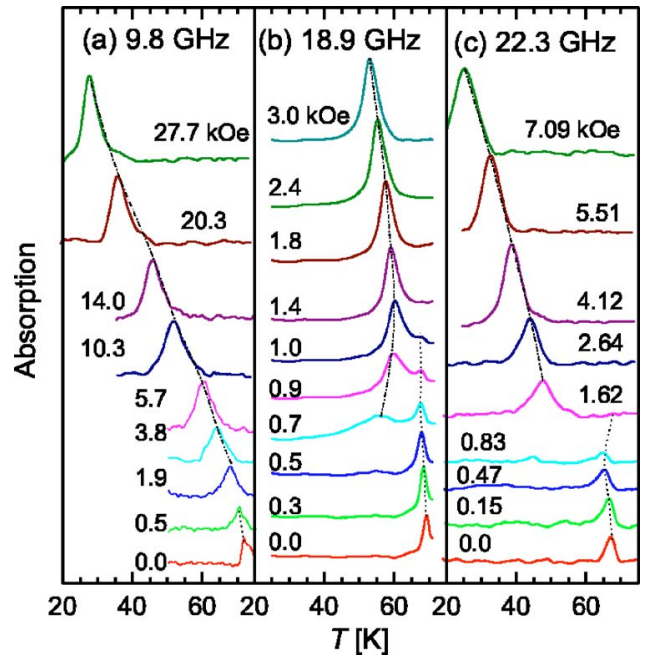

FIG. 2. (Color online) The resonance curves observed at 9.8 (a), 18.9 (b), and 22.3 (c) GHz. The temperature is swept down from far beyond $T_{c}$ after setting magnetic fields. At $9.8 \mathrm{GHz}$ (a), the separation of the LTM and HTM cannot be identified although the line shape changes from asymmetric to symmetric in a low-field region below $0.5 \mathrm{kOe}$. At higher frequencies than $18.9 \mathrm{GHz}$, a sharp HTM and relatively broad LTM are split well and easily identified.

a function of temperature as well as magnetic field.

Focusing on the HTM, it behaves in a very unusual manner as seen in Fig. 1(a), for example. In zero magnetic field a sharp resonance with a symmetric line shape with respect to temperature was clearly observed. Since this resonance corresponds to the zero-field mode argued previously, the temperature dependence of the zero-filed plasma frequency $\omega_{p}(T)$ can be described by the two-fluid model assuming the conventional Drude model..$^{37}$

In a finite parallel magnetic field below approximately $1 \mathrm{kOe}$, the HTM begins to shift slightly to lower tempera-

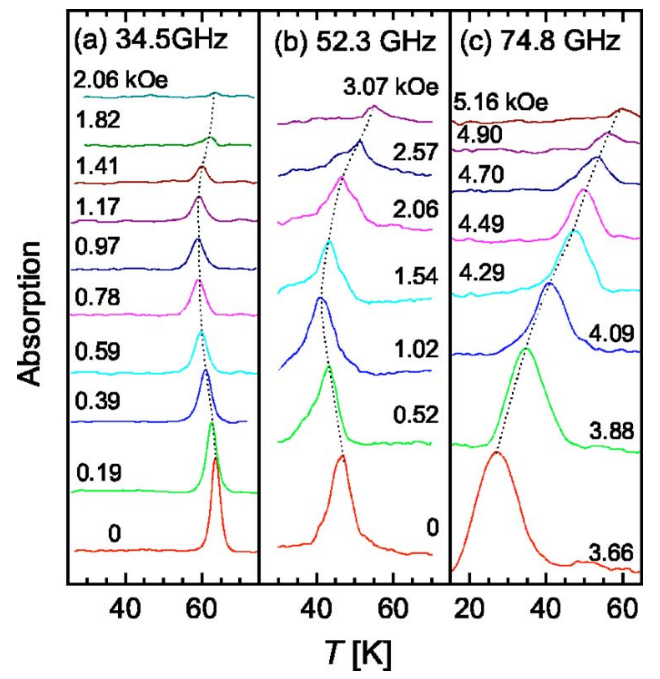

FIG. 3. (Color online) The resonance curves observed at 34.5 (a), 52.3 (b), and 74.8 (c) $\mathrm{GHz}$ by sweeping the temperature from above $T_{c}$. In this high-frequency region, only the HTM was observed. The resonance drastically shifts to lower temperatures and broadens as $\omega$ is increased. At $74.8 \mathrm{GHz}$, the HTM was obtained only above $3 \mathrm{kOe}$ without zero-field resonance. 


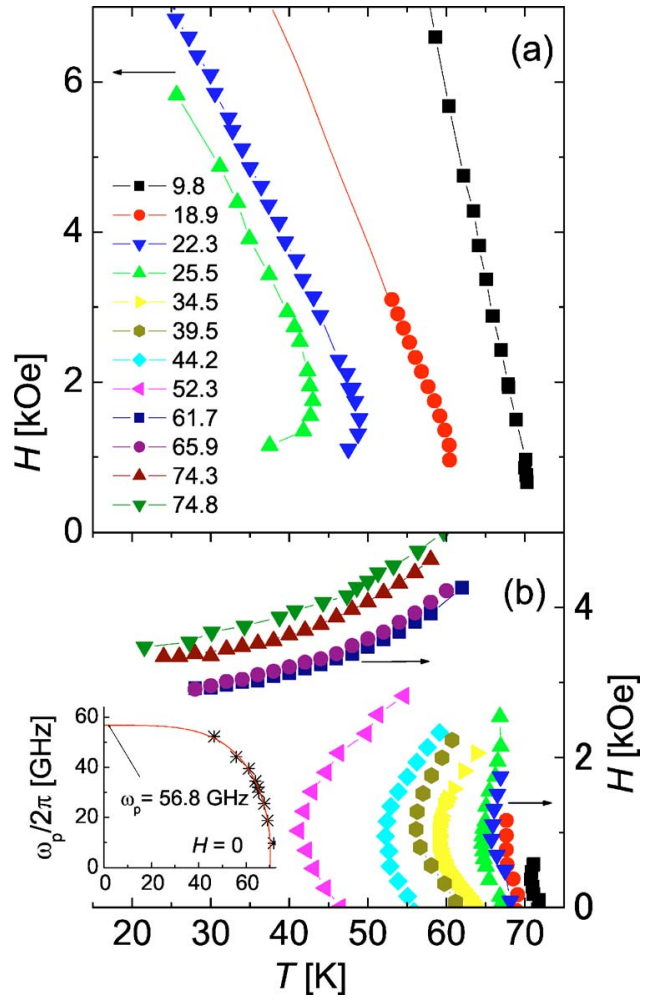

FIG. 4. (Color online) (a) The resonance field-temperature plot for the LTM resonance obtained at 9.8, 18.9, 22.3, and 25.5 GHz. (b) The resonance field-temperature plot for the HTM resonance obtained at 9.8, 18.9, 22.3, 25.5, 34.5, 39.5, 44.2, 52.3, 61.7, 65.9, 74.3 , and $74.8 \mathrm{GHz}$ from right to left. The same symbols represent data obtained at the same frequencies. Inset: the $\omega_{p}$ vs $T$ plot at zero field. Asterisks are experimental data, and the solid line is a curve fitted to the two-fluid model.

tures. With further increase of the magnetic field, however, it turns to shift backward to higher temperatures at around $0.8 \mathrm{kOe}$ as obviously seen in Fig. 1(a) [this behavior is more clearly displayed in Fig. 4(b), where the data obtained at many frequencies measured are presented for the LTM]. The strong absorption intensity of this HTM in zero field quickly decreases as the field is applied. The linewidth in terms of temperature is wider in these finite fields than in the zero field.

It has been well established that $\omega_{p}$ is always suppressed by application of perpendicular magnetic fields, ${ }^{22,23}$ because the Josephson current due to coherence effect between layers is a decreasing function of magnetic field. ${ }^{21}$ This requires that in higher magnetic fields the resonance temperature must be lower in order to gain the fraction of Josephson current which is reduced by the perpendicular magnetic fields. It seems that this is not the case in the Josephson plasma resonance in parallel fields to the layers and this phenomenon certainly requires a new explanation.

Such an unusual turnover behavior just mentioned above cannot be explained either without making an unusual assumption that the phase coherence between layers would increase further with further increase of the parallel magnetic field approximately above $0.8 \mathrm{kOe}$ or without introducing entirely new mechanisms for the Josephson plasma reso- nance in the parallel magnetic field. It is worth mentioning that this increasing tendency of the temperature dependence of $H_{\text {res }}$ toward higher temperatures seems never to go beyond $T_{c}$ in zero field, although it goes beyond the plasma resonance temperature $T_{0}$ in zero field at the frequency $\omega$ as seen in Fig. 4(b). It fades away quickly just below $T_{c}$. This surprising result may allow us to speculate that the HTM is more stable in temperatures with the formation of the Josephson lattice state than without Josephson lattices. This unusual behavior has never been observed in the Josephson plasma resonance in perpendicular magnetic fields studied earlier and is certainly a new phenomenon only observable in parallel magnetic fields.

In parallel magnetic fields, consecutive occupation of the JV's in between the $\mathrm{CuO}_{2}$ layers is expected to be realized along the $c$ axis as a function of increasing magnetic field, preserving an isosceles triangular symmetry according to the theoretical calculations. ${ }^{38}$ This formation of $\mathrm{JV}$ lattices would cause the oscillatory coherent Josephson current, which accordingly possesses the wave vector $\boldsymbol{k}$ associated with the periodicity of the JV's, and would provide the resonance at even higher temperatures in a finite field than that in zero field according to the periodic JV arrangement.

In the intermediate field region between 1.3 and $2.2 \mathrm{kOe}$ both the HTM and LTM can be observed at the same magnetic field as seen in Fig. 1(a). For instance, the data at $1.69 \mathrm{kOe}$ indicate the existence of both the HTM and LTM with $25.5 \mathrm{GHz}$ at $(1.69 \mathrm{kOe}, 66.2 \mathrm{~K})$ and $(1.69 \mathrm{kOe}$, $43.0 \mathrm{~K})$, respectively. This feature is observed only within a certain frequency region between 18 and $30 \mathrm{GHz}$, and only one resonance line can be found except this frequency range. This observation of two resonance modes indicates a peculiarity in the Josephson plasma resonance in $\boldsymbol{H} \| a b$ unlike the one for $\boldsymbol{H} \| c$, where only one resonance line, as far as the longitudinal mode is concerned, was observed to monotonically decrease with both increasing temperature and magnetic field.

The resonance curves obtained by FS measurements are displayed in Fig. 1(b). As the temperature is just below $T_{c}$ $(=70.2 \mathrm{~K})$ a sharp resonance begins to appear $\left(T_{0} \simeq 68 \mathrm{~K}\right)$ from the zero-field limit. The resonance rapidly grows with decreasing temperature. As a few degrees Kelvin below $T_{0}$, two resonance line can be distinguished: one at a lower field is stronger in intensity than the other one which lies at a higher fields and at the foot of the resonance of the stronger line (pointed by arrows). This two-resonance feature can only be found in $62 \lesssim T \$ 66 \mathrm{~K}$, below which the resonance suddenly changes its character with broadening and with highly the hysteretic nature as seen in Fig. 1(b). The temperature range is wider at higher frequencies. For example, it extends to $14 \mathrm{~K}$ at $52.3 \mathrm{GHz}$, above which frequency the two resonance feature can no longer be observed.

As seen in Fig. 1(b) the absorption curves show a considerable hysteretic behavior for the field being swept up and down, especially below $45 \mathrm{~K}$. This hysteretic feature is not appreciable at high temperatures above $60 \mathrm{~K}$ whereas it is most prominent in the intermediate temperature region between 10 and $45 \mathrm{~K}$. It is noticed that there are several small resonancelike peaks at the lower-field side in addition to the main large resonance. However, this anomaly as well as even 
in the main peaks depends strongly on the field sweeping direction and the sweeping rate. Furthermore, it must be mentioned that some faint peaks are observed in between the HTM and LTM in the FS mode [at $52 \mathrm{~K}$ in Fig. 1(b), for example], where there is no resonance in the TS mode because of the gap between the HTM and LTM as seen in Fig. 1(a). The peaks are, perhaps, nonresonant absorptions due to the nonequilibrium effect of the JV state because the peak fields are almost equal to the fields where the LTM is observed at the highest temperatures. Therefore, we do not consider these faint peaks in the FS measurement hereafter.

\section{B. Frequency dependence}

\section{Low-frequency region $(\omega / 2 \pi<30 \mathrm{GHz})$}

The typical resonance curves at 9.8, 18.9, and $22.3 \mathrm{GHz}$ obtained by TS measurements are shown in Figs. 2(a)-2(c), respectively. At the lowest frequency of $9.8 \mathrm{GHz}$, only one resonance line is observed. The resonance line shape changes from asymmetric to symmetric with increasing field below $0.5 \mathrm{kOe}$, indicating a precursor effect of splitting into the HTM and LTM already at this frequency region. In sharp contrast to this, the resonance splitting is clearly observed at 18.9 GHz as shown in Fig. 2(b), where the LTM resonance and the HTM resonance appear below and above $64 \mathrm{~K}$, respectively. The HTM resonance becomes weak in intensity and is quickly smeared with increasing field, and finally disappears at $1.0 \mathrm{kOe}$, while the LTM resonance appears from the low-temperature side above about $0.7 \mathrm{kOe}$, then shifts to the higher-temperature side, and then turns back to lower temperatures with increasing field with little change in the line shape up to $3 \mathrm{kOe}$. The larger separation between the LTM and HTM resonances at higher frequencies indicates that the LTM has a different frequency dependence from the HTM. The integrated $H-T$ diagrams for most frequencies for both the LTM and HTM are shown in Fig. 4 by plotting the resonance temperatures as a function of field.

The resonance curves obtained in FS measurements for various temperatures at the same frequencies shown in Fig. 2 are shown in Fig. 5. All resonance peaks displayed here belong to the LTM except for the ones indicated by the inverted triangles. The resonance peaks of the LTM in increasing field lie at a lower field than the ones in decreasing field, and the hysteresis observed in increasing and decreasing magnetic fields becomes more significant at lower temperatures and in lower fields. In comparison with the TS data, the resonance peaks obtained in the case of decreasing field coincide with the peaks in the TS measurements rather well, in which more homogeneous Josephson vortex state is expected to occur.

It is very unlikely that a complete lock-in vortex state is realized without any pancake vortices in the actual experimental condition, because the calculated angle to be allowed to form the lock-in state by taking into account of the sample geometry (the interlayer distance of $12 \AA$ and the length of the sample of $0.8 \mathrm{~mm}$ ) would be of the order of $10^{-4} \mathrm{deg}$ or less, which is much smaller than the experimental angular resolution. Furthermore, from the x-ray parallel-beam double-crystal method, it has been known that our sample used for the present experiment has a width of the rocking

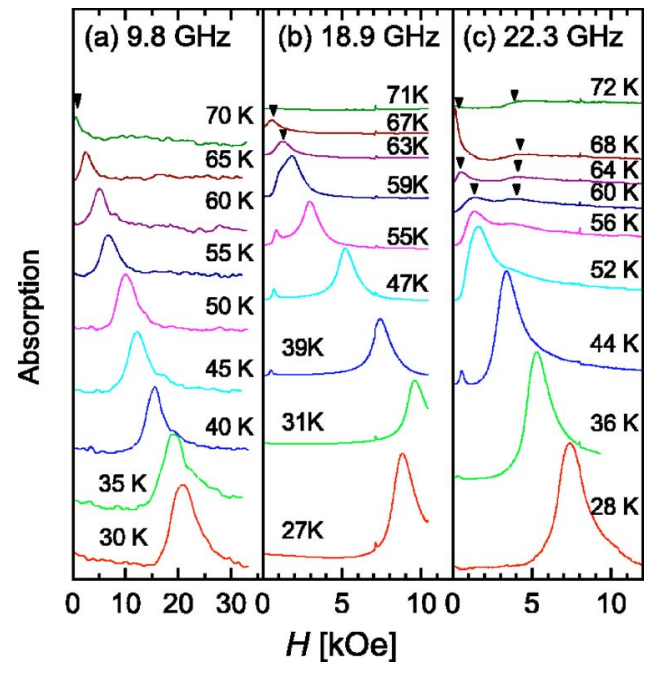

FIG. 5. (Color online) The resonance curves at 9.8 (a), 18.9 (b), and 22.3 (c) $\mathrm{GHz}$ by sweeping the magnetic field to $H=0$ after stabilizing temperatures at the maximum fields. The resonance peaks pointed by inverted triangles correspond to the HTM resonance.

curve of the order of $0.025^{\circ}$. This means that the crystallographic imperfectness of our sample, especially the parallelism of the $\mathrm{CuO}_{2}$ layers, gives an actual limitation to the measurements. Therefore, we think that the JV state is always accompanied by some amount of the remaining pancake vortices. In such a situation the vortex crossing lattice state would be formed as first suggested by Koshelev, ${ }^{39}$ where the interaction between Josephson vortices and pancake vortices may be attractive. Since the pancake vortices are pinned strongly at low temperatures as seen in the large hysteresis in magnetization, the JV system would be compelled to be pinned by the pancake vortices through an attractive interaction. At the present stage of knowledge we do not know to what extent this pinning effect is important in the Josephson plasma phenomena except for the hysteretic effect while the magnetic field is swept.

However, it is important to note that the complete JV state can be established in a smaller sample with dimensions less than $10 \mu \mathrm{m} \times 10 \mu \mathrm{m}$ in the $a b$ plane. This is evidenced by the angular dependence measurement of the JV flow resistance which shows a sudden rise at an angle within $1^{\circ}$ or so from the $a b$ plane, depending strongly on the sample size and the intensity of the applied magnetic field. ${ }^{40}$

\section{High-frequency region}

Figures 3(a)-3(c) depict resonance absorption curves at $34.5,52.3$, and $74.8 \mathrm{GHz}$, respectively, by TS measurements at various fixed magnetic fields. In this higher-frequency region, only the HTM is observed. As seen from Fig. 3, it is indispensable to note that the HTM at 34.5 and $52.3 \mathrm{GHz}$ has zero-field resonance, while it does not have zero-field resonance at $74.8 \mathrm{GHz}$. This is because the HTM in the low-field region quickly shifts to the low-temperature side and disappears above approximately $57 \mathrm{GHz}$. This can be seen more clearly in Fig. 4(b), where the HTM is shown in the $H-T$ 


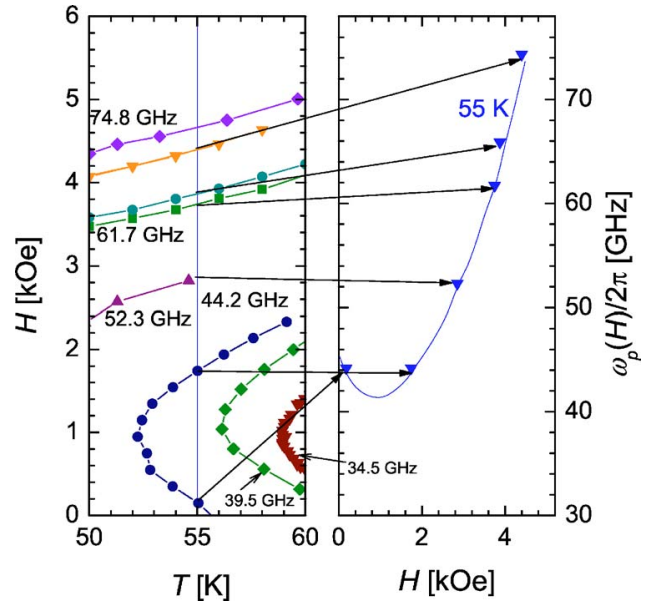

FIG. 6. (Color online) The reploted $H_{\mathrm{res}}-T$ diagram from the resonance field-temperature plot shown in Fig. 4 to the $\omega$ - $H$ diagram at various frequencies at $55 \mathrm{~K}$ as an example.

plot. This zero-field resonance occurs only at a particular temperature $T_{0}$ at the corresponding $\omega$. The $\omega$ dependence of $T_{0}$ has been reported previously ${ }^{37,41}$ and is explained by using a simple two-fluid model with a temperature-independent scattering rate. ${ }^{37} \mathrm{By}$ extrapolating this temperature dependence of the plasma frequency $\omega_{p}(T)$ to $T=0$ as shown in the inset of Fig. 4(b), the inherent (zero-temperature) plasma frequency $\omega_{p}(0) / 2 \pi$ is estimated to be $56.8 \mathrm{GHz}$, which yields $\lambda_{c}=217 \mu \mathrm{m}$ with $\epsilon=15$. This value of $\lambda_{c}$ is quite reasonable for underdoped BSCCO with $T_{c}$ of $70.2 \mathrm{~K}$ and is used to quantify the field dependence of the HTM in the later sections.

In the frequency range below but near $\omega_{p}(0)$, the dramatic turnover behavior of the resonance temperature is observed as seen in Fig. 4(b). The Josephson plasma resonance at low fields below $1 \mathrm{kOe}$ shifts to the lower-temperature side and then turns back to higher temperatures above $1 \mathrm{kOe}$ as the magnetic field is increased. A typical example is shown in the $H-T$ plot in the left panel of Fig. 6 in the case of $\omega / 2 \pi$ $=44.2 \mathrm{GHz}$, where $T_{0}=55.7 \mathrm{~K}$ and the shift in temperature amounts to about $3.5 \mathrm{~K}$ below $T_{0}$ at about $1 \mathrm{kOe}$, then the resonance peak sharply turns over toward the hightemperature side, and reaches to $59 \mathrm{~K}$, which is even more than $3 \mathrm{~K}$ higher than $T_{0}$. The intensity of the resonance becomes weaker and weaker with increasing field and the resonance finally fades away. As the frequency is increased, all the curves including $T_{0}$ move towards low temperature as shown in the $H-T$ plot in Fig. 4(b). However, it is interesting to note that the disappearing temperature of the resonance after the turnover lies in the temperature region between 55 and $65 \mathrm{~K}$ even above the zero-field resonance temperature $T_{0}$. This surprising behavior is very different from our understanding of the Josephson plasma resonance for $\boldsymbol{H} \| c$, where the plasma frequency should decrease with application of a magnetic field and with increasing temperature as a result of the suppression of the interlayer coherence $\left\langle\cos \varphi_{l, l+1}(H, T)\right\rangle$ due to the fluctuation of pancake vortices as formulated in Eq. (1).

At frequencies above $\omega_{p}(0)$, there is no longer the resonance line at $H=0$ but only one resonance in a finite field in

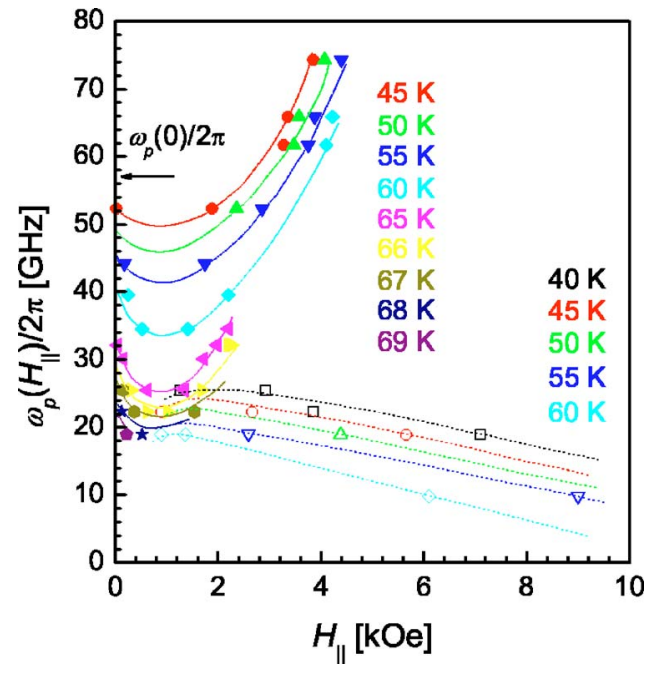

FIG. 7. (Color online) The frequency-field diagram for the HTM and LTM. Solid symbols denote the HTM at 45, 50, 55, 60, 65, 66, 67,68 , and $69 \mathrm{~K}$, and open symbols denote the LTM at 40, 45, 50, 55 , and $60 \mathrm{~K}$ from top to bottom. Solid and dotted curves are guides for the eyes.

the HTM. As shown in Fig. 3(c), the resonance line can be observed only above about $3 \mathrm{kOe}$, and the resonance occurs at higher temperatures in higher magnetic field until reaching the limiting temperature of about $60 \mathrm{~K}$ around $5 \mathrm{kOe}$. This means that the excitation energy of the HTM becomes higher at higher temperatures. Since the superconducting phase coherence should become weaker and weaker at higher temperatures, this increasing excitation energy of the Josephson plasma in the HTM needs a new expression if it is enhanced further by overcoming the reducing temperature effect. As shown in Fig. 4(b), three more data sets obtained at different frequencies are included.

\section{Overall features}

In order to construct the $\omega_{p}-H$ diagram, which is a direct indication of the $H_{\|}$effect on the excitation modes, the following procedure is applied: Since the most of experiments have been done in the TS mode at a fixed magnetic field at a certain $\omega$ and the $H-T$ diagram was constructed as shown in Fig. 4 , one should convert this to the $\omega_{p}-H$ plot by taking the resonance points from corresponding $\omega$ at various temperatures as shown in Fig. 6. The resonance frequency is plotted as a function of magnetic field for nine different temperatures in Fig. 7. It is noted that the plots in Fig. 7 were extracted from resonance field versus temperature plots at all measured frequencies (partly not shown in Fig. 4).

It is clear that there are two different type of resonance modes with very different field-dependent characteristic features: one corresponds to the HTM, which starts with $\omega_{p}(0)$ at zero field; then, the frequency grows monotonically and almost linearly after passing a shallow minimum in the $\omega_{p}$ vs $H$ diagram as the field is increased. On the contrary to this, the LTM shows a weak initial increasing behavior with increasing magnetic fields and then turns to the gradual decreasing behavior after passing a broad maximum. This LTM seems to be disappear at the zero-field limit. 


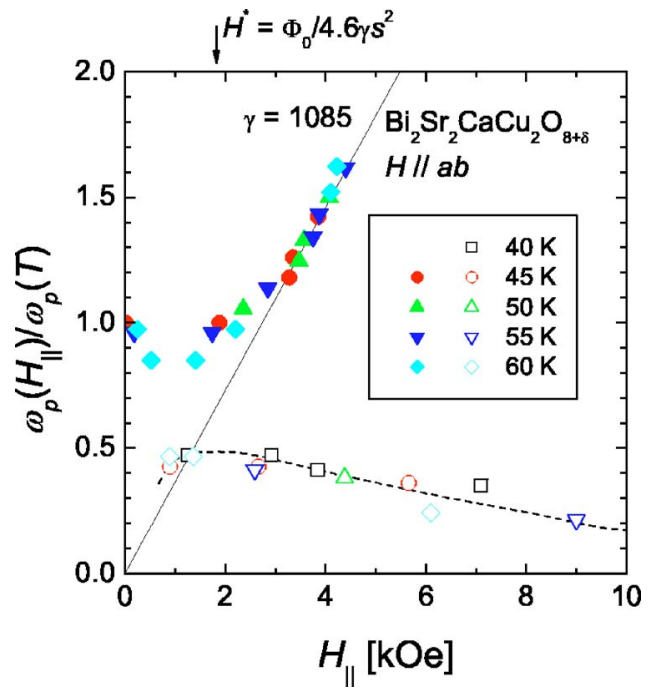

FIG. 8. (Color online) $\omega_{p}\left(H_{\|}\right) / \omega_{p}(T)$ of both modes at 40, 45, 50,55 , and $60 \mathrm{~K}$ is shown. Solid and open symbols denote the HTM and LTM, respectively, and a solid line is given by Eq. (2) with $\gamma=1085$.

As mentioned above, the HTM deviates from linear behavior and tends to have a minimum at $0.5-1.0 \mathrm{kOe}$, and then merges with the zero-field Josephson plasma mode. Since this HTM is directly connected to the zero-field Josephson plasma mode, the HTM is confirmed to be the Josephson plasma mode in the JV state in a parallel external magnetic field. It is noted that the initial decrease of $\omega_{p}\left(H_{\|}\right)$ is stronger at higher temperatures. On the other hand, the LTM exhibits a broad peak at around $1.5 \mathrm{kOe}$ and then tends to decrease again with decreasing field. It is important to note that this LTM disappears at the limit of zero magnetic field. This experimental fact implies strongly that the existence of JV's is crucial to the LTM.

In Fig. $8, \omega_{p}\left(H_{\|}\right)$normalized by the zero-field plasma frequency at a given temperature derived from the inset of Fig. 4(b) is shown for five typical temperatures in Fig. 7. As seen in Fig. 8 it is interesting to point out that both the HTM and LTM are scaled well to two single curves by using $\omega_{p}(T)$.

\section{Universality of two excitation modes}

The microwave measurements have been performed in a similar manner in other crystals with higher doping levels (U2 and OP samples). The essential features described above were unchanged: the characteristic two modes HTM and LTM exist with qualitatively similar temperature as well as magnetic field dependencies. A typical example of the experimental data are shown in Figs. 9(a) and 9(b) for an underdoped U2 sample and for an optimally doped OP sample, respectively. Comparing these data with the data shown in Figs. 2 and 3, one can easily find that the separation between the HTM and LTM for U2 is smaller than that of U1 at a similar frequency. For example, the data in Fig. 9(a) (at $34.4 \mathrm{GHz}$ ) can be compared with the data in Fig. 3(a) (at $34.5 \mathrm{GHz})$ and are rather similar to the those obtained at $22.3 \mathrm{GHz}$ shown in Fig. 2(c). Assuming that these two data

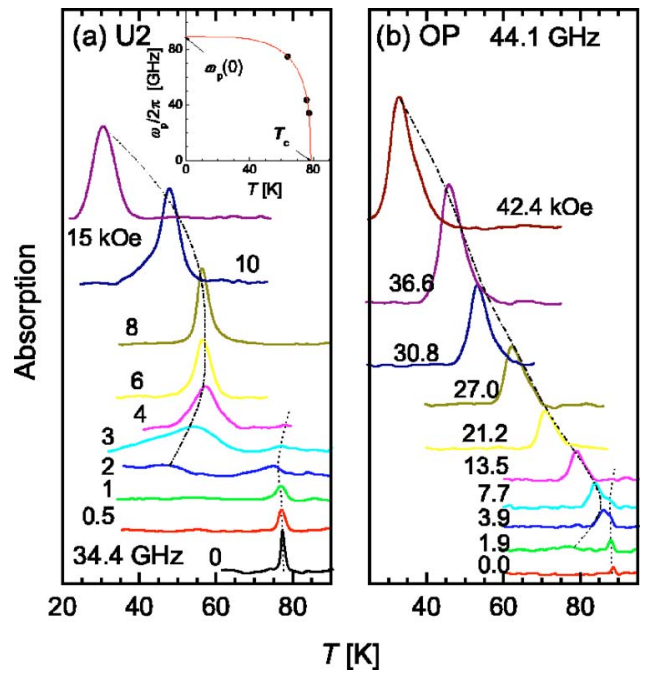

FIG. 9. (Color online) The resonance data obtained (a) at $34.6 \mathrm{GHz}$ in $\mathrm{U} 2\left(T_{c}=76.8 \mathrm{~K}\right)$ and (b) at $44.1 \mathrm{GHz}$ in OP $\left(T_{c}\right.$ $=91.0 \mathrm{~K})$. Two resonances and the temperature gap are found in both samples. Separation of two modes at 4 kOe in U2 (a) is similar as the data at $22.3 \mathrm{GHz}$ and $1.6 \mathrm{kOe}$ in $\mathrm{U} 1$ [Fig. 2(c)]. The inset of (a) shows the temperature dependence of the zero-field plasma frequency in U2. Dotted lines trace the HTM resonance and are guides for the eyes, which shows turnover behavior as well as the HF mode in $\mathrm{U} 1$.

sets obtained at 22.3 and $34.5 \mathrm{GHz}$ provide the same ratio of $\omega / \omega_{p}(0)$ to each sample, $\omega_{p}(0) / 2 \pi$ for $\mathrm{U} 2$ can be derived as $88.1 \mathrm{GHz}$. This estimated value of $\omega_{p}(0)$ is consistent and agrees rather well with the value extrapolated by the temperature dependence of the zero-field Josephson plasma frequency $\omega_{p}(T)$ as shown in the inset of Fig. 9(a), although the number of experimental points are only a few.

In the case of the OP sample, typical experimental data are shown in Fig. 9(b) at $44.1 \mathrm{GHz}$, where no clear splitting of the HTM and LTM is observed. Applying the similar scaling in this case (for $18.9 \mathrm{GHz}$ in U1), a value of $\omega_{p}(0) / 2 \pi$ $\approx 130 \mathrm{GHz}$ is derived. This value is close to the one obtained for an optimally doped Bi2212 single crystal. ${ }^{41}$ Thus we suggest that the resonance splitting can be noticeably observed at $\omega \gtrsim \omega_{p}(0) / 3$ and the magnitude of the $T$ gap is larger for $\omega / \omega_{p}(0)$ being closer to unity. In contrast, $\omega$ is much smaller than $\omega_{p}(0)$, and the LTM merges to HTM as observed at $9.8 \mathrm{GHz}$ in $\mathrm{U} 1$, resulting in a single branch of the $H$-T plot.

In previous studies, Matsuda et al. ${ }^{30}$ and Tsui et al. ${ }^{31}$ have reported that the Josephson plasma resonance field has a sharp decrease near $\theta=0(\boldsymbol{H} \| a b)$. In particular, Matsuda et $a l$. found the disappearance of resonance in an underdoped crystal $\left(T_{c}=87 \mathrm{~K}\right)$ through measurements of the angular dependence of the FS data and argued the existence of a collective mode of the Josephson vortex lattice which lies above the experimental microwave frequency $(\omega / 2 \pi=45 \mathrm{GHz})$ at zero field and increases with parallel magnetic fields on the basis of a theory by Bulaevskii et al. ${ }^{32}$ Judging from our results, this vanishing of the resonance observed by Matsuda et al. can be interpreted differently because their sample is underdoped crystal and the measurement was done at $36 \mathrm{~K}$. Their experimental conditions seem to be in the gap region 


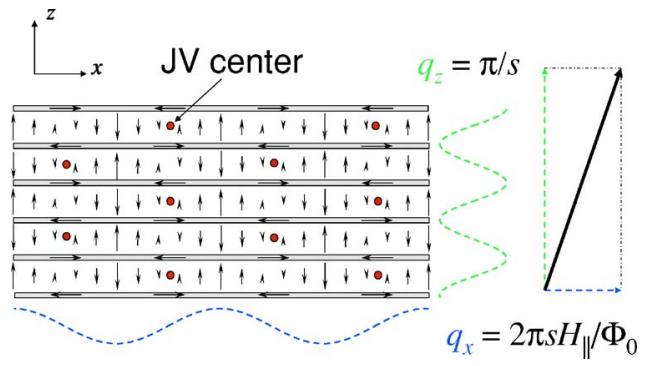

FIG. 10. (Color online) A schematic picture of the propagation of the tilted plasma wave. The interlayer phase difference along the $a b$ plane and $c$ axis is spatially modified as shown by the dotted and dashed waves, respectively. The propagation vector of the plasma wave comprised of the transverse component $q_{x}$ and the longitudinal component $q_{z}$ is depicted as a thick arrow.

between the HTM and LTM. Furthermore, according to their results the disappearance was not observed in an optimally doped crystal $\left(T_{c}=89.5 \mathrm{~K}\right)$. This also seems to be consistent with the systematics which we found here although they attributed it to the misalignment of the magnetic field. From an experimental point of view, no matter what the theoretical interpretation may be, the observed dramatic change of the Josephson plasma resonance near $\theta=0$ is strongly suggestive for the excitation of the new phase collective modes in intrinsic Josephson junctions.

\section{ORIGIN OF EXCITATION MODES}

\section{A. High-temperature mode}

As discussed by Fetter and Stephen in single junctions, ${ }^{27}$ propagating Josephson plasma waves are strongly modified by the JV's, resulting in the Josephson plasma mode having a linear field dependence according to the linear increase of the reciprocal lattice vector of the JV array, $k_{H}=2 \pi s H_{\|} / \Phi_{0}$, where $\Phi_{0}$ is the flux quantum. This indicates that a periodic arrangement of JV's is crucial to have the propagating Josephson plasma mode. We first assume that JV's penetrate all block layers and form isosceles triangular lattice with the lattice constants along the $c$ axis and $a b$ plane being $2 s$ and $\Phi_{0} / s H_{\|}$in the high- $H_{\|}$region, respectively, as shown in Fig. 10 , although for layered superconductors the argument has not been completely settled yet. A theoretical calculation by Ichiok $\mathrm{a}^{42}$ predicts that such a dense JV lattice can be realized above $H^{*}=\sqrt{3} \Phi_{0} / 8 \gamma s^{2}$, which gives $1.8 \mathrm{kOe}$ for $\gamma=1085 \mathrm{ob}-$ tained from $\lambda_{c}=217 \mu \mathrm{m}$ and $\lambda_{a b}=2000 \AA$. This value is rather in good agreement with the magnetic field, where the HTM begins to tend to the linear relation and the LTM has a broad maximum. Hence, it is reasonable to think that the HTM above $H^{*}$ may be the Josephson plasma resonance mode in the dense JV lattice.

Considering a Josephson plasma wave traveling in the isosceles triangular JV lattice, the $\boldsymbol{k}$ vector of the Josephson plasma mode corresponds to the primitive reciprocal lattice vector of the JV lattice, $\boldsymbol{q}$, which consists of the constant $c$ axis component $q_{z}=\pi / s$ and the $H_{\|}$proportional $a b$ plane component $q_{x}=2 \pi s H_{\|} / \Phi_{0}$, as depicted in Fig. 10. Here, $y$ and $z$ axes are taken parallel to the JV and $c$ axis, respec- tively, and $x$ is taken perpendicular to the $y$ and $z$ axes. Thus, Josephson plasma waves can be excited at all possible wave vectors as of the mixture of the longitudinal plasma with $k_{z}$ $=q_{z}$ and the transverse plasma with $k_{x}=q_{x}$. The plasma frequency is determined by the dispersion relation along $\boldsymbol{q}$ lying between the longitudinal and transverse plasma modes and increases with magnetic field because of the linear increase in $q_{x}$. It should be noted that the dispersion is very close to the longitudinal dispersion for $q_{x} \ll q_{z}$.

Bulaevskii et al. have proposed a formulation of the plasma resonance in a layered superconductor on the basis of the single-junction model, ${ }^{32}$ and Koshelev and Machida have derived excitation spectra by considering the longitudinal coupling effect. ${ }^{34,35}$ In the high-field limit, where all block layers are occupied by JV's forming an isosceles triangular lattice, the peak frequency of the dissipation spectrum, corresponding to the resonance peak, as a function of parallel magnetic field is formulated as

$$
\frac{\omega_{p}\left(H_{\|}\right)}{\omega_{p}(0)}=\frac{\pi \gamma s^{2}}{\Phi_{0}} H_{\|} .
$$

Using $\gamma=1085$, this equation provides an excellent agreement with the experimental data above $3 \mathrm{kOe}$ at all temperatures, as shown in Fig. 8. It is also consistent that the linear field dependence of the HTM is violated below $H^{*}$. We therefore conclude that the linear increase in $\omega_{p}\left(H_{\|}\right)$of the HTM is due to an increase in $q_{a b}$ of the JV lattice, while $q_{c}$ is fixed on $\pi / s$ because of the intrinsic pinning. This result suggests that the excited plasma frequency can be easily controlled by adjusting the magnetic fields parallel to the layers even above $\omega_{p}$.

\section{B. Low-temperature mode}

The LTM lies at $\omega \lesssim \omega_{p} / 2$ and slightly decreases with $H_{\|}$ as displayed in Fig. 8. The LTM in crystals with higher doping tends to appear at higher frequencies although the whole $\omega_{p}-H$ diagram has not been established yet. In under doped YBCO, a similar excitation mode was observed around $\omega_{p} / 2$ with slight negative $H_{\|}$dependence and is referred to the $\alpha$ mode. ${ }^{43}$ The $\alpha$ mode cannot be observed after zero-field cooling, suggesting the considerable influence of pinning of the JV's on the collective oscillation mode. This may also be the case in Bi2212 as shown in Fig. 1(b) and as well as in Fig. 5, where a large hysteretic effect is observed. Assuming that our LTM corresponds to their $\alpha$ mode, the low-lying collective mode should strongly depend on $\omega_{p}$, which is a material parameter and given by the strength of the Josephson coupling between $\mathrm{CuO}_{2}$ bilayers.

It is well known that the lowest collective mode of a JV system is the vortex sliding mode with $k_{x}=0 .{ }^{27}$ The sliding mode becomes gapless in an ideal system without pinning and couples with a dc homogenous electric field perpendicular to the superconducting layers. Since the pinning of Josephson vortices cannot completely be removed in actual samples, the lowest mode should have a nonzero frequency. In a junction with a periodic pinning for instance, the Josephson critical current depends periodically on $x .^{32}$

Since the hysteresis obtained by the FS measurements indicates that the JV pinning strongly affects the LTM, we 
once discussed the pinned vortex sliding scenario as a possible origin of the LTM. ${ }^{44}$ However, our finding that the LTM tends to become higher in more highly doped samples with higher $\omega_{p}$ cannot be explained by this scenario, because it is not natural that the collective mode hardened by the pinning effect can be scaled by $\omega_{p}$. Therefore, the vortex sliding model is not suited and not likely for the origin of the LTM in the framework of conventional knowledge and needs a new explanation.

In order to reveal the origin of the LTM, it is required to introduce an intrinsic stacking effect of intrinsic Josephson junctions because previous theoretical models giving the vortex sliding mode are based on the single-junction model, where the correlation of the JV lattice along the $z$ axis is neglected. The vortex sliding mode in layered superconductors which we have in mind means coherent vortex motion parallel and perpendicular to the layers with $k_{x}=k_{z}=0$. Here, we may consider a collective mode with $k_{x}=0$ but $k_{z} \neq 0$ at a finite frequency. Considering an antiphase oscillation of JV arrays between adjacent junctions with $k_{z}=\pi / s$ and $k_{x}=0$, for instance, the oscillation is a sharing motion between adjacent JV arrays as a result of dynamic phase oscillations not only between adjacent layers but also next-adjacent layers, so that the frequency of the oscillation can be considered to be scaled by $\omega_{p}$ because the interaction between JV's in adjacent junctions is to be governed by the Josephson coupling as the first approximation.

Very recently, Koyama reported theoretical calculations on the Josephson plasma resonance in the JV lattice. ${ }^{33}$ After numerical calculations on analytically derived equations, a collective mode which lies about $\omega_{p}(0) / 2$ in the low-field limit and slightly decreases with increasing field was obtained. He argues that the collective mode is considered as an antiphase oscillation mode of JV's which originates from the strong charge coupling between junctions, and it is also responsible for the longitudinal Josephson plasma mode. This picture seems to be most appropriate as a candidate for the origin of the LTM although no direct experimental proof has been obtained.

For deeper understandings of the LTM, further theoretical and experimental investigations are needed in the lowfrequency region. We think that an investigation of the JV flow branch in the current-voltage characteristics along the $c$ axis is a complementary experiment to elucidate the JV behavior in the layered superconductors in the low-frequency region.

\section{CONCLUSIONS}

We have clearly identified two microwave excitation modes with a temperature-dependent gap in parallel magnetic fields in single crystal $\mathrm{Bi}_{2} \mathrm{Sr}_{2} \mathrm{CaCu}_{2} \mathrm{O}_{8+\delta}$. It is shown that the strong-coupling effect between the Josephson plasma and JV lattice is responsible for these two modes. The highfrequency mode is attributed to the Josephson plasma mode propagating along the primitive reciprocal lattice vector of the JV lattice. Making use of this interplay, we may be able to explore electromagnetic waves in the frequency range between the superconducting gap and $\omega_{p}$. The low-frequency mode is attributed to the new collective mode which has never been observed before and seems to be unique for layered superconductors. It is considered that the Josephson vortex collective oscillation mode with finite $k_{z}$ contributes to the phase oscillations, although a quantitative full understanding is has not been obtained.

\section{ACKNOWLEDGMENTS}

We would like to thank Drs. M. Machida, A. Koshelev, T. Koyama, H. Matsumoto, K. M. Kojima, and N. F. Pedersen for their fruitful discussions and critical comments. This work was supported by Grant-in-Aid for Young Scientists (B), No. 14740201, 2002-2003, and 21st Century Center of Excellence (COE) Program at University of Tsukuba under MEXT, Japan.
*Electronic address: kakeya@ims.tsukuba.ac.jp

${ }^{1}$ P. W. Anderson, Lectures on the Many-Body Problem (Academic Press, New York, 1964), Vol. 2, p. 113.

${ }^{2}$ A. J. Dahm, A. Denenstein, T. F. Finnegan, D. N. Langenberg, and D. J. Scalapino, Phys. Rev. Lett. 20, 859 (1968).

${ }^{3}$ N. F. Pedersen, T. F. Finnegan, and D. N. Langenberg, Phys. Rev. B 6, 4151 (1972).

${ }^{4}$ R. Kleiner, F. Steinmeyer, G. Kunkel, and P. Müller, Phys. Rev. Lett. 68, 2394 (1992).

${ }^{5}$ R. Kleiner and P. Müller, Phys. Rev. B 49, 1327 (1994).

${ }^{6}$ M. Tachiki, T. Koyama, and S. Takahashi, Phys. Rev. B 50, 7065 (1994).

${ }^{7}$ L. N. Bulaevskii, M. P. Maley, and M. Tachiki, Phys. Rev. Lett. 74, 801 (1995).

${ }^{8}$ T. Koyama and M. Tachiki, Phys. Rev. B 54, 16183 (1996).

${ }^{9}$ M. Machida, T. Koyama, and M. Tachiki, Phys. Rev. Lett. 83, 4618 (1999).
${ }^{10}$ N. F. Pedersen and S. Sakai, Phys. Rev. B 58, 2820 (1998).

${ }^{11}$ S. Sakai and N. F. Pedersen, Phys. Rev. B 60, 9810 (1999).

${ }^{12}$ M. Machida and S. Sakai, Phys. Rev. B 70, 144520 (2004).

${ }^{13}$ Y. Matsuda, M. B. Gaifullin, K. Kumagai, K. Kadowaki, and T. Mochiku, Phys. Rev. Lett. 75, 4512 (1995).

${ }^{14}$ K. Kadowaki, I. Kakeya, M. B. Gaifullin, T. Mochiku, S. Takahashi, T. Koyama, and M. Tachiki, Phys. Rev. B 56, 5617 (1997).

${ }^{15}$ Y. Ohashi and S. Takada, Phys. Rev. B 59, 4404 (1999).

${ }^{16}$ K. Tamasaku, Y. Nakamura, and S. Uchida, Phys. Rev. Lett. 69, 1455 (1992).

${ }^{17}$ S. Sakamoto, A. Maeda, T. Hanaguri, Y. Kotaka, J. Shimoyama, K. Kishio, Y. Matsushita, M. Hasegawa, H. Takei, H. Ikeda, and R. Yoshizaki, Phys. Rev. B 53, R14749 (1996).

${ }^{18}$ S. Tajima, J. Schützmann, S. Miyamoto, I. Terasaki, Y. Sato, and R. Hauff, Phys. Rev. B 55, 6051 (1997).

${ }^{19}$ T. Shibauchi, M. Sato, A. Mashio, T. Tamegai, H. Mori, S. 
Tajima, and S. Tanaka, Phys. Rev. B 55, R11977 (1997).

${ }^{20}$ L. N. Bulaevskii, V. L. Pokrovsky, and M. P. Maley, Phys. Rev. Lett. 76, 1719 (1996).

${ }^{21}$ A. E. Koshelev, Phys. Rev. Lett. 77, 3901 (1996).

${ }^{22}$ T. Shibauchi, T. Nakano, M. Sato, T. Kisu, N. Kameda, N. Okuda, S. Ooi, and T. Tamegai, Phys. Rev. Lett. 83, 1010 (1999).

${ }^{23}$ I. Kakeya, R. Nakamura, T. Wada, and K. Kadowaki, Physica C 362, 234 (2001).

${ }^{24}$ A. E. Koshelev, L. N. Bulaevskii, and M. P. Maley, Phys. Rev. B 62, 14403 (2000).

${ }^{25}$ K. Kadowaki, I. Kakeya, K. Kindo, S. Takahashi, T. Koyama, and M. Tachiki, Physica C 293, 130 (1997).

${ }^{26}$ P. Lebwhol and M. J. Stephen, Phys. Rev. 163, 376 (1967).

${ }^{27}$ A. L. Fetter and M. J. Stephen, Phys. Rev. 168, 475 (1968).

${ }^{28}$ R. E. Eck, D. J. Scalapino, and B. N. Taylor, Phys. Rev. Lett. 13, 15 (1964).

${ }^{29}$ M. D. Fiske, Rev. Mod. Phys. 36, 221 (1964).

${ }^{30}$ Y. Matsuda, M. B. Gaifullin, K. Kumagai, K. Kadowaki, T. Mochiku, and K. Hirata, Phys. Rev. B 55, R8685 (1997).

${ }^{31}$ O. K. C. Tsui, S. P. Bayrakci, N. P. Ong, K. Kishio, and S. Wa- tauchi, Phys. Rev. B 56, R2948 (1997).

${ }^{32}$ L. N. Bulaevskii, D. Domínguez, M. P. Maley, and A. R. Bishop, Phys. Rev. B 55, 8482 (1997).

${ }^{33}$ T. Koyama, Phys. Rev. B 68, 224505 (2003).

${ }^{34}$ A. E. Koshelev and I. Aranson, Phys. Rev. B 64, 174508 (2001).

${ }^{35}$ A. E. Koshelev and M. Machida (private comminucation).

${ }^{36}$ I. Kakeya, K. Kindo, K. Kadowaki, S. Takahashi, and T. Mochiku, Phys. Rev. B 57, 3108 (1998).

${ }^{37}$ K. Kadowaki, I. Kakeya, T. Wakabayashi, R. Nakamura, and S. Takahashi, Int. J. Mod. Phys. B 14, 547 (2000).

${ }^{38}$ X. Hu and M. Tachiki, Phys. Rev. Lett. 85, 2577 (2000).

${ }^{39}$ A. E. Koshelev, Phys. Rev. Lett. 83, 187 (1999).

${ }^{40}$ I. Kakeya et al. cond-mat/0503498.

${ }^{41}$ M. B. Gaifullin, Y. Matsuda, N. Chikumoto, J. Shimoyama, K. Kishio, and R. Yoshizaki, Phys. Rev. Lett. 83, 3928 (1999).

${ }^{42}$ M. Ichioka, Phys. Rev. B 51, 9423 (1995).

${ }^{43}$ K. M. Kojima, S. Uchida, Y. Fudamoto, and S. Tajima, Phys. Rev. Lett. 89, 247001 (2002).

${ }^{44}$ I. Kakeya, T. Wada, M. Machida, and K. Kadowaki, Physica C 378-381, 437 (2002). 